\title{
Écriture-dévoilement ou la méthodologie historique au service de la littérature : Mes soixante ans de Constance de Salm
}

\author{
Maryam SHARIF \\ Kharazmi University \\ maryam.sharif@khu.ac.ir \\ ORCID : 0000-0001-6395-0255
}

\section{Resumen}

Cuando una obra literaria no pretende mezclar realidad y ficción, sino escribir lo real con las operaciones cognitivas que esto implica, la literatura se convierte en una herramienta para explicar y comprender el mundo. Mes Soixante ans de Constance de Salm es un texto literario que, a pesar del discurso que ejemplifica, se asemeja a una obra histórica. Tras analizar la naturaleza híbrida de la obra con la ayuda de diferentes elementos del método histórico -como la posición del "yo" del investigador, la movilización de referencias y evidencias, la estructuración cronológica del documento- y del comparatismo, nos proponemos demostrar la inteligibilidad científica del poema.

Palabras clave: Escritura del yo. Estudios interdisciplinares. Escritora francesa. Historia. Siglo XIX.

\section{Résumé}

La littérature devient un outil d'explication-compréhension du monde lorsqu'une œuvre littéraire ne vise pas à mélanger le factuel et la fiction mais à écrire le Réel avec les opérations cognitives que cela suppose. Mes soixante ans de Constance de Salm est un texte constitutivement littéraire qui pourtant, par le discours qu'il exemplifie, s'apparente à un ouvrage historique. Après avoir analysé le caractère hybride de l'œuvre, à l'aide de divers éléments de la méthodologie historique comme la position du « je » de chercheur, la mobilisation des sources et des preuves, la structuration chronologique du document ainsi que le comparatisme, nous allons démontrer l'intelligibilité scientifique du poème.

Mot-clés: Écriture du moi. Études interdisciplinaires. Écrivaine française. Histoire. XIX siècle.

\begin{abstract}
When a work of literature doesn't aim to mix fact with fiction but aims to write the reality with the cognitive operations that it's assumed, the literature becomes a tool of explanation and comprehension of the world. Mes soixante ans is a literary text which can present
\end{abstract}

* Artículo recibido el 1/03/2020, aceptado el 13/04/2020. 
itself as a historical work by using the type of discourse that it illustrates. After analyzing the hybrid character of the work, the position of the "I" of the method, the movement of the sources and the evidence, the chronological structuring of the document as well as comparatism, are the various elements of the historical methodology; with the help of which the historical intelligibility of the poem is then demonstrated.

Keywords: Narrating the Self. Interdisciplinary study. French women writer.. XIX ${ }^{\text {th }}$ century.

\section{Introduction}

Si la production de connaissances est le propre d'une démarche scientifique, un texte littéraire dont le sujet serait sociologique, anthropologique, psychanalytique ou historique, peut être considéré comme une œuvre scientifique. C'est moins la visée de l'énoncé que la méthodologie de l'énonciation qui oriente l'horizon d'attente du chercheur en littérature et peut rendre un texte, dont la fonctionnalité principale est esthétique, porteur de savoir. Si au premier regard le roman, par son désintéressement relatif à l'égard du monde réel semble difficilement se livrer à une lecture objective et scientifique, les textes comme l'autobiographie, les mémoires, le carnet de route, etc. intéressent le lecteur non seulement par leur contenu, mais aussi par la connaissance qu'ils engendrent dans le domaine des sciences dites sociales.

Qu'en est-il d'un récit de vie en vers? D’une part, de quelque manière qu'on définisse la forme poétique, "un poème, comme dit Gérard Genette (2004 : 7), est toujours une ouvre littéraire, parce que les traits formels (variables) qui le marquent comme poème sont, de manière non moins évidente, d'ordre esthétique ». D'autre part, un récit de vie dans lequel la frontière entre l'objectivité, l'historicité et la fictionalité n'est point étanche (Thion Soriano-Mollà et al., 2016:7), est censé produire un contenu fondé essentiellement sur le réel ; or la fiction est le caractère thématique par excellence de la littérarité.

Néanmoins le réel, depuis le premier $\mathrm{XIX}^{\mathrm{e}}$ siècle, est un thème de la grande littérature comme l'Histoire au sens latin de « connaissance acquise par l'enquête » est une manière de penser. Ainsi, le rhématique d'un texte, comme le veut Genette (2004 : 7), c'est-à-dire le type du discours qu'un texte exemplifie, pourrait en faire un objet à fonction non esthétique mais scientifique : producteur de connaissance. Un texte dont le contenu est la non-fiction et dont la forme est la poésie est-il de la littérature ou de l'Histoire ? Par et dans le discours qu'il engendre, aide-t-il à mieux connaître le monde?

La théorie du texte-dévoilement d'Ivan Jablonka (2014) semble pouvoir répondre à la question : dès lors qu'une méthodologie est perceptible dans l'élaboration d'un texte constitutivement littéraire, on est en face d'une œuvre aussi scientifique qu'esthétique : 
[La littérature du réel est] caractérisée avant tout par son rapport au monde. Elle n'est pas définie par son objet (les « faits ») ni par son manque (la «non-fiction»), mais par son désir de compréhension, sa potentialité explicative. En d'autre terme, si littérature du réel il y a, elle est plus cognitive que réaliste. Théorie d'une écriture-dévoilement : au lieu d'imiter le réel ou d'inventer des histoires, la littérature peut tenter de dire vrai au sujet du monde (Jablonka, 2014 : 312).

Ainsi, l'autobiographie, les mémoires ou les journaux intimes, en raison de leur tentative d'explication-compréhension du monde, sont des textes qui se prêtent par excellence à une lecture interdisciplinaire historico-littéraire. Car réfléchir sur le passé, futil même récent, et établir le lien entre le présent de l'écriture et le passé exige une méthode :

Écrire sous le signe de la mémoire, c'est aller du présent vers le passé, du moins vers certains moments ou événements du passé, en les invoquant ou en les convoquant dans le présent. Ce passé, on peut l'embrasser et le présentifier ou rencontrer l'irrémédiable distance qui nous en sépare, ou faire les deux. Pour mener cette opération, le recours à une démarche historienne a toute sa place comme technique d'enquête (Hartog, $2013: 28)$.

Mes soixante ans, ou mes souvenirs politiques et littéraires (1833) de Constance de Salm est l'exemple type d'un texte hybride où une intelligibilité historique se fait voir à travers ses 1272 vers. Son étude démontrerait comment la réminiscence du passé est imprégnée d'argumentation. Citons d'emblée l'article paru en Çédille, intitulé "Mes soixante ans y sus referentes políticos y literarios: las memorias en verso de Constance de Salm » (Romera Pintor, 2015b), lequel offre déjà l'analyse de ce texte et en fait largement connaître les enjeux historiques et politiques afin de démêler l'intelligibilité scientifique du poème. On vise, ici, le même objectif mais pour y atteindre, on entreprend une approche autre et une méthodologie différente.

Le triple "je» de recherche du sujet écrivant (de position, d'enquête, d'émotion), l'usage des sources et des preuves ainsi que le comparatisme sont des méthodes d'historien auxquelles l'écrivaine a fréquemment et inconsciemment recours pour produire sa version de l'Histoire. Après avoir présenté le texte en vue de clarifier son hybridité, nous y décèlerons la démarche d'historienne pour conclure sur non seulement la viabilité mais également la nécessité des études interdisciplinaires appliquées aux textes littéraires pour en révéler les aspects objectifs et scientifiques.

Constance de Salm fait partie de ces nombreuses femmes écrivains du passé qui, publiées et reconnues de leur vivant, ont laissé peu de traces dans l'histoire littéraire. Ce n'est depuis les années quatre-vingt, que ces dernières sont au cœur d'un 
mouvement de réhabilitation et de reconnaissance académique ${ }^{1}$ et (dans une moindre mesure) publique. L'entreprise commence d'ailleurs à révéler ses résultats : en ce qui concerne Constance de Salm, Éliane Viennot (2016: 5516) tient compte de «la grande poétesse Pipelet $»^{2}$ dans une position égale à Germaine de Staël étant en soi un canon littéraire et, à la librairie, son roman se trouve traduit en différentes langues (anglais, allemand, espagnol, catalan, italien).

Née le 7 novembre 1767 à Nantes et décédée le 13 avril 1845 à Paris, Salm a vécu plus de trois quarts de siècle : élevée sous l'Ancien Régime, mariée l'année de la Révolution avec Monsieur Pipelet de Leury, divorcée sous le Consulat (ou à la fin du Directoire), remariée sous l'Empire avec le comte de Salm Dyck, élevée au rang de princesse sous la Restauration (par le roi de Prusse) elle appartient ainsi aussi bien au siècle des Lumières qu'au premier XIX ${ }^{\mathrm{e}}$ siècle.

À la suite de son mariage avec M. Pipelet, elle s'installe à Paris, fait désormais partie des milieux lettrés; Marie-Joseph Chénier la surnomme "la muse de la raison ». En 1795, elle est la première femme admise au Lycée des Arts. Elle a eu une longue carrière littéraire avec des œuvres assez variées. Ses premières publications datent de 1785 dans le Journal général de France et dans l'Almanach des Grâces sous son nom de jeune fille incomplet : Constance de $\mathrm{TH}^{* * *}$. La poésie constitue la plus grande partie de ses œuvres (épître, éloges, boutades, discours, souvenirs en vers) et son Épître aux femmes, réponse à la querelle des femmes poètes suscitée par Écouchard Lebrun dans les pages de la Décade philosophique durant l'année 1797 est aujourd'hui reconnue comme un des textes fondateurs du féminisme avant-garde ${ }^{3}$.

${ }^{1}$ En 1989 sont publiés l'ouvrage de Geneviève Fraisse La Muse de la raison : la démocratie exclusive et la différence des sexes, qui présente Salm comme une pionnière féministe, et l'article de Christine Planté "Constance Pipelet : la Muse de la Raison et les despotes du Parnasse », qui étudie sa poésie. Depuis lors les travaux universitaires s'enchaînent. Le colloque de Toulouse (2009) consacré à l'ensemble de l'œuvre et de la carrière de l'écrivaine et dont les actes sont publiés dans les Cabiers Roucher AndréChénier $\left(\mathrm{n}^{\circ}\right.$ 29) fut un tournant dans les études sur Salm. Constance de Salm est aujourd'hui une auteure incontournable dans les études consacrées aux femmes, auteures et autres, du premier XIX siècle. Outre celles et ceux dont les articles sont publiés dans les Cahiers Roucher-André Chénier (comme Seth, 2010a) et qui ne cessent d'enrichir la littérature critique sur Salm, l'auteure a trouvé sa place aussi bien dans l'histoire des femmes (Viennot, 2016 ; Christine le Bozec, 2019) que dans l'histoire de l'art (cf. Fend, Hyde \& Lafont, dir., 2012). Des chapitres des livres lui ont été consacrés (cf. Reid, 2012). Les universitaires francophones ont aussi abordé les divers aspects de son ouvre : les travaux de Á. M. Romera-Pintor en est l'exemple significatif, notamment son ouvrage Constance de Salm y la modernidad de su discurso feminista. Epistolas y otros escritos (1767-1845) (2015a). L'écrivaine est aussi le sujet de différents mémoires et thèses, par exemple : Luzon (1997), Mattos (2007) ou Sharif (2014).

${ }^{2}$ Le corpus auquel Viennot donne référence est écrit et publié avant le divorce de l'écrivaine quand elle s'appelait donc Constance Pipelet.

${ }^{3}$ Quelques-uns de ses poèmes sont publiés dans des anthologies dès années soixante du XX $\mathrm{XX}^{\mathrm{e}}$ siècle : Huit siècles de Poésie féminine. Anthologie (de Jeanine Moulin, Paris, Seghers, 1963, 117-120), Le Grief des femmes. Anthologie des textes féministes du Moyen Âge à la seconde République (de Maïté Albistur et Daniel Armogathe, Paris, Hier et demain, 1978, 193-194), The Defiant Muse. French Feminist poems 
Elle a aussi écrit deux pièces de théâtre : la première, en 1794, est Sapho, une "tragédie mêlée de chants " et mise en musique par Martini (1741-1816), qui fut un succès et eut environ cent représentations au théâtre des amis de la patrie ( $c f$. Letzter, 1999; Adelson \& Letzter, 2000; Sharif, 2015 ou Perazzolo, 2018). Sa seconde tentative était avec un drame appelé Amitié et Imprudence (1799) qui semble avoir suscité des critiques et qui fut retiré après une seule représentation. Salm a écrit des éloges de savants contemporains, parfois à leur propre demande (Joseph de Lalande, astronome 1732-1807), ce qui atteste encore de sa réputation dans les milieux savants. Elle a aussi écrit et lu dans des séances de la société des savants ou du lycée des arts, des rapports sur l'œuvre de ses contemporains ${ }^{4}$. Elle a aussi publié les fragments d'un essai de comparaison : Les Allemands comparés aux Français (cf. Berenguier, 2017 et 2018). La correspondance de l'écrivaine rend aussi compte non seulement de la continuité de ses réflexions littéraires et politiques mais également de ses échanges intellectuelles avec ses contemporain.e.s des milieux de lettres et de sciences 5 .

Elle a également publié un roman: Vingt-quatre heures d'une femme sensible (1824) réédité, avec succès, en 2007 chez Phébus et en 2012 chez Flammarion dans la collection «Étonnant Classique ». Sa traduction dans diverses langues ainsi que son adaptation sur la scène ont fait connaitre l'auteure en dehors des milieux acadé-

from the Middle East to Present (de Domna C. Stanton, The Feminist Press at CUNY, 1986, 108-128) ou Femmes poètes du XIX siècle. Une Anthologie (dirigée par Christine Planté, Lyon, PUL, 2010 [1998], 51-65) et qui font aussi paraître des extraits de ses épîtres ou ses poésies diverses. Désormais il semble difficile d'ignorer Salm, qui prend aussi place dans des anthologies non-exclusivement consacrés aux femmes poètes (Anthologie de la poésie française, t. II, éd. de Martine Bercot, Michel Collot et Catriona Seth, Paris, Gallimard, "Bibliothèque de la Pléiade », 2000, 414-422).

${ }^{4}$ Son Rapport sur un ouvrage du $C^{e n}$ Théremin, intitulé : De la condition des femmes dans une République (Paris, impr. de Gillé, an VIII ) est largement commenté par Viennot (2016).

${ }^{5}$ La correspondance de Salm a largement intéressé les chercheurs et les chercheuses. Les premières recherches universitaires consacrées à Salm ont notamment abordé cette correspondance : Robert Bied, "Le rôle d'un salon littéraire au début du XIX siècle : les amis de Constance de Salm " (Revue de l'Institut Napoléon, 133, 1977, 121-160 ; Robert Bied \& Henri Coulet, «Une partie d'échecs de Constance de Théïs " (Le jeu au XVIII siècle, Aix-en-Provence, Édisud, 1976, 141-156). Les années quatrevingt-dix sont marquées par les travaux anglo-saxons : Elizabeth Colwill, "Law of nature / rights of genius : the drame of Constance de Salm "(Going public: Women and publishing in early modern France, Cornell University Press, 1995, 224-242) ; "Epistolary passions : friendship and the literary public of Constance de Salm" (Journal of Women's History, 12/3, 2000, 39-68). La monographie d'Ellen McNiven Hine, Constance de Salm, her influence and her circle in the aftermath of the French Revolution : a mind of no common order (New York, Peter Lang, 2012) se fonde aussi sur la correspondence de Salm, laquelle est aussi étudiée dans le cadre de deux thèses : celle de Marie-Thérèse Raguet, Correspondance de Constance de Salm (1795-1811). Édition critique (Université Aix-Marseille I, 2008) et celle de Eve-Marie Lampron, Entre cohésions et divisions : les relations entre femmes auteures en France et en Italie (1770-1840) (Université de Montréal, 2012). Une base de données de l'abondante correspondance de Salm est aussi mise en ligne par l'Institut Historique Allemand (disponible sur: https://dhiha.hypotheses.org/1336) qui avait aussi organisé en 2011 un colloque autour de ce sujet. 
miques ${ }^{6}$. Un recueil de pensées sur différents sujets dont une partie sera publiée à titre posthume, clôt la carrière artistique d'une femme qui se vante elle-même d'être restée toujours la même tout au long de sa vie : raisonneuse, analytique, attachée aux idéaux des Lumières et aux principes littéraires classiques.

\subsection{L'œuvre}

Mes soixante ans, les souvenirs en vers de Salm, est un témoignage sur la vie d'une écrivaine qui définit son entreprise littéraire en étroite relation avec l'Histoire. L'œuvre se présente comme une chronologie de l'histoire politique de la France: commençant par le moment glorieux de la Révolution, elle parcourt la Terreur, l'Empire, la Restauration pour finir avec la Monarchie de Juillet. S'entremêlent avec l'histoire politique les événements de la vie littéraire de l'écrivaine, c'est-à-dire les moments où, en tant que femme auteur, elle présente une œuvre au public et voit accepté et applaudi le fruit de son travail (cf. Crogiez Labarthe : 2010).

Le texte est accompagné d'un sommaire (Salm, 1842: 266) qui affirme la double appartenance de Mes soixante ans au privé et au public et expose les titres des ses différentes séquences, résumant ainsi les sujets et les matières traités. Cette classification respecte les grandes dates de l'histoire de la France à partir des années qui s'écoulent depuis la Révolution de 1789. Elle adopte en effet une pratique devenue courante parmi les mémorialistes de l'époque :

L'individu se découvre sujet historique; il s'observe, déroule son existence comme une durée intérieure et publique à la fois ; ce faisant, il sort le temps public de son abstraction et lui donne l'épaisseur d'un vécu. Rien ne signale mieux ces phénomènes que la pratique de la datation comparée à laquelle les mémorialistes ont fréquemment recours : depuis 1789, le morcellement du devenir politique en périodes découpées strictement par la scansion de révolutions, coups d'État et revers militaires fournit un calendrier commun qui impose à chacun de réfléchir sur la matière dont est faite la durée propre de son existence (Zanone, 2006 : 141).

L'exemple d'une telle datation se trouve à cette époque chez Madame la duchesse d'Abrantès qui intitule son ouvrage: les Mémoires de Madame la duchesse d'Abrantès, Souvenirs historiques sur Napoléon: la Révolution, le Directoire, le Consulat, l'Empire et la Restauration (1831). Constance de Salm n'agit pas différemment : Révolution, Terreur, Empire, Guerres de 1813, 1814 et 1815, Restauration, séparation des provinces de Rhin, Congrès de Vienne et Révolution de 1830 répartissent les différentes séquences de Mes soixante ans et leur servent de titres. Trente-sept séquences de longueurs différentes scandent ainsi le texte. Si on exclut l'introduction et

${ }^{6}$ Salm romancière a également tirée l'attention de différents chercheurs, notons entre autres Paquin (1998), Seth (2010b) ou Planté (2012). 
la conclusion, seules douze séquences abordent, d'une manière directe ou indirecte, les œuvres de l'écrivaine ou ses réflexions littéraires et philosophiques; les vingt-trois autres relatent les événements historiques. Par conséquent, le texte est marqué par l'articulation entre le factuel et l'intime, donnant un récit de vie qui flotte entre mémoires et autobiographie.

Selon Philippe Lejeune (1998 : 10), l'autobiographie est « un récit rétrospectif en prose que quelqu'un fait de sa propre existence, quand il met l'accent principal sur sa vie individuelle, en particulier sur l'histoire de sa personnalité ». Or, non seulement Mes soixante ans est écrit en vers mais la vie personnelle de l'écrivaine n'y occupe qu'une place mineure. L'on n'y trouve aucun éclaircissement sur la famille de l'écrivaine ni sur ses racines, aucune explication au sujet de son enfance ou de sa formation, aucune révélation à propos de ses relations avec autrui. S'agit-il d'une " autobio-graphie» au sens où l'entend George Gusdorf (1991: 245) lorsqu'il dit: "l'autobiographie ne devrait plus être considérée comme un genre littéraire parmi les autres, mais comme un mode de lecture applicable aux œuvres littéraires les plus diverses " ? Ou s'agit-il plutôt de mémoires, genre en plein essor durant les années qui précèdent et qui suivent la publication de Mes soixante ans? Entre 1815 et 1845 "quelque six cent titres de Mémoires paraissent, dont à peu près quatre cent cinquante inédits consacrés à l'époque récente (les autres étant des rééditions de Mémoires des siècles antérieures)»(Zanone, 2006 : 15). Dans des mémoires, selon Lejeune (1998: 11), "l'auteur se comporte comme un témoin : ce qu'il a de personnel, c'est le point de vue individuel, mais l'objet du discours est quelque chose qui dépasse de beaucoup l'individu, c'est l'histoire des groupes sociaux et historiques auquel il appartient ». Alors que l'autobiographie vise la vie privée de l'individu, les mémoires se penchent sur la vie publique, et si dans le premier c'est le moi intime de l'écrivain qui est exposé, dans le second c'est le moi social qui transmet ses expériences.

Pourtant est-il possible, dans ce début du XIX ${ }^{\mathrm{e}}$ siècle, de parler du " moi » toujours haïssable depuis Blaise Pascal ? La répugnance à s'attarder sur un récit intime en plein tumulte de l'histoire mène parfois les auteurs à rejeter toute tentative d'écrire leurs mémoires, comme Benjamin Constant qui, à la suite de la publication de l'ouvrage de Louis Bignon (1771-1841), Histoire de France depuis le 18 brumaire jusqu'en 1812 fait publier ses Souvenirs historiques de février à juin 1830 dans la Revue de Paris. Il écrit :

Écrire des mémoires me répugne. Les hommes les plus spirituels, les plus distingués par le tact et la mesure, sont, à leur insu, entraînés par ce genre de travail à parler beaucoup de ce qui les concerne personnellement. Je ne les en blâme pas; je lis sans ennui ce qu'ils nous apprennent de leur naissance, de leur éducation, de leurs amours, même de leurs maladies; mais je ne m'intéresse pas assez à moi pour en occuper si longuement les autres, et il me serait impossible de dire comme un général 
d'ailleurs célèbre (Dumouriez) : «Tandis que la France était en feu, j'étais enrhumé au fond de la Normandie " (Constant, $1992: 84)$.

Parfois pour se justifier d'écrire et de publier leurs souvenirs personnels, les écrivains revendiquent leur utilité publique. Germaine de Staël (1996 : 207) commence ses Dix années d'exil par ces mots :

Ce n'est point pour occuper le public de moi que j'ai résolu de raconter les circonstances de dix années d'exil ; les malheurs que j'ai éprouvés, avec quelque amertume que je les aie sentis, sont si peu de chose au milieu des désastres publics dont nous sommes témoins, qu'on aurait honte de parler de soi, si les événements qui nous concernent n'étaient pas liés à la grande cause de l'humanité menacée.

Relier son histoire personnelle à celle du monde sert ainsi de justification pour avoir pris la parole à la première personne. En l'occurrence, certains écrivains essaient d'effacer leur « je » tout en relatant leur propre histoire. Madame de Staël (1996 : 208) écrit qu'" [elle se] flatte de [se] faire souvent oublier en racontant [sa] propre histoire ". Plus qu'un discours sur soi, l'écriture des mémoires est ainsi un geste historique de témoignage. S'entremêlent ainsi l'écriture de soi et celle du monde :

Par convention littéraire et sociale, le «moi» est encore haïssable au début du XIX ${ }^{\mathrm{e}}$ siècle ; mais dans le même temps, la curiosité pour l'histoire contemporaine saisie sous forme de témoignages vécus devient obsédante à la suite de la Révolution et de l'Empire. Il faut composer avec les deux exigences - ou alors se taire comme Constant (Zanone, 2006 : 24).

Ainsi dans ce début du $\mathrm{XIX}^{\mathrm{e}}$ siècle, pour une écrivaine attachée aux valeurs classiques, voire opposée au romantisme naissant ${ }^{7}$, le caractère impersonnel des événements dignes d'être rapportés semble tellement aller de soi que lorsque Salm se trouve obligée de suggérer quelques traits de sa vie privée, elle se sent contrainte d'expliquer ce choix :

J'ai cru même devoir y rappeler, lorsque mon sujet m'a paru l'exiger, les différentes situations dans lesquelles le sort m'a placée, et, en général, m’y livrer librement à toutes mes inspirations, et m'y montrer dans mon caractère et dans mes sentiments comme dans mes opinions (Salm, 1842 : II).

L'expression de ses sentiments sert à clarifier les conditions concrètes de son écriture et ne relève jamais d'une situation privée. La seule fois où elle suggère une étape de sa vie

\footnotetext{
${ }^{7}$ Son poème "Sur le romantique », paru en 1824, est un manifeste contre le romantisme (Salm, 1842,
} II : 197). 
privée, c'est en raison de l'impact de ce changement sur sa vie de femme de lettres. Il s'agit de son second mariage qui l'introduisit dans la noblesse d'Empire : elle pouvait désormais observer de près les événements politiques et le caractère de l'homme au pouvoir. Dans une perspective d'autojustification, elle essaie alors d'opérer un raccord entre l'Histoire, son entreprise littéraire et sa vie privée. En outre, Salm qualifie Mes soixante ans de "simple exposé " et d' "aperçu rapide" : employant ainsi des adjectifs qui réduisent le texte à un résumé laconique, elle tente de minimiser l'audace de prendre la parole à la première personne.

Par ailleurs, dans l'avertissement de son texte, tout en exposant son projet, Salm écrit "Je n'ai jusqu'à présent parlé de moi dans aucun de mes ouvrages ", phrase qui engendre l'attente d'un récit autobiographique où elle donnerait des renseignements sur sa vie intime mais cette attente est vite déçue, puisqu'elle poursuit ainsi : "J'ai éprouvé le besoin de me retracer ces temps si beaux et si extraordinaires que j'ai traversés, de me rendre compte de l'influence presque continuelle qu'ils ont eu sur ma longue carrière, $[\ldots] »$. Et elle continue :

Ces souvenirs, $[\ldots]$ sont enfin un tableau fidèle de ce que j'ai vu, de ce que j'ai pensé, de ce qui, pendant plus de quarante ans, a occupé ou agité mes esprits. Celui qui les aura lus m'aura vue vivre; il aura parcouru avec moi cette longue série d'événements qui rendent ce siècle si célèbre, et, si je ne me trompe, il aura une idée juste de l'impression qu'ils produisaient sur nous, et de l'esprit qui nous animait dans ces temps de grandeur et gloire, si différents de ceux que nous voyons aujourd'hui (Salm, 1842 : II).

L'accent est mis sur l'ordre du monde et sur le vécu historique de toute une génération. Salm affirme sa double position comme objet et comme sujet et à ce titre désire transmettre son expérience et ses jugements. Elle est d'abord en position d'objet, puisque ce sont les événements extérieurs qui influencent son comportement; et sa position de sujet ne dérive que de son rôle de témoin face à l'histoire. Pourtant, existe-elle une opposition substantielle entre l'individu et la société ? D'après Pierre Bourdieu (1982 : 38) le social est inscrit dans l'individuel :

[Le Principe de l'action historique] ne réside ni dans la conscience ni dans les choses, mais dans la relation entre deux états du social, c'est-à-dire entre l'histoire objective dans les choses, sous forme d'institutions, et l'histoire incarnée dans les corps, sous la forme de ces dispositions durables que j'appelle habitus.

L'habitus qui résulte de la socialisation de l'auteur permettrait non seulement une meilleure compréhension de l'œuvre mais également celle du processus de la production littéraire. Ce processus ressemble à une quête artistique lorsque les différentes opérations cognitives, conscientes ou inconscientes, s'accumulent pour retracer les expériences que les corps séparés ont partagées. 
Ce partage se révèle d'ailleurs dans la conversion, dans la dernière citation, du " je " à "nous ". Le moi ne se tient pas jusqu'au bout de l'exposé et cède la place à "nous ", l'individuel fait place au collectif : conversion qui renforce la prétention de produire un récit qui sort des frontières du moi pour aborder le monde. Cette conversion montre aussi que trancher entre la subjectivité et l'objectivité est inconcevable. Ce qu'on retrouvera presque deux siècle après, explicitement chez Annie Ernaux dont les œuvres se construisent sur un "je " qui cède, certes avec une claire conscience méthodologique, la place à " elle ", " on " ou " nous. " Dans Écrire la vie, elle explique: "J'ai toujours écrit à la fois de moi et hors de moi, le "je" qui circule de livre en livre n'est pas assignable à une identité fixe et sa voix est traversée par les autres voix, parentale, sociales, qui nous habitent" (Ernaux, 2011 : 7-8). De même, dans l'excipit des Années, elle écrit: "Aucun "je" dans ce qu'elle voit comme une sorte d'autobiographie impersonnelle - mais "on" et "nous" -, comme si à son tour, elle faisait le récit des jours d'avant " (Ernaux, $2008: 252)$.

Les formules de Genette (2004: 68) pour schématiser les relations entre l'Auteur, le Narrateur et le Personnage confirment également cette double appartenance du récit à l'intime et au factuel :

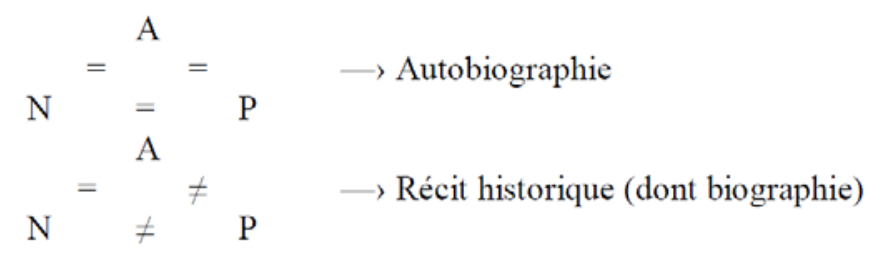

Parfois les trois angles d'auteur, de narrateur et de personnage sont dans une relation d'égalité : ils constituent donc une autobiographie; parfois, l'auteur et le narrateur sont le même mais le personnage n'est ni auteur ni narrateur mais un " nous " ou un " on " qui s'apparente à « je "; dans ce cas, le texte est un récit historique.

Si c'est la proportion entre l'intime et le social qui permet de décider du genre littéraire de cette œuvre, Mes soixante ans relève des mémoires, et d'ailleurs c'est bien ainsi que l'appelle Constance de Salm elle-même qui dit : " mes mémoires moraux, ». C'est l'histoire vécue et sentie par elle ou comme dit Gusdorf (1991 : 252) « la chronique personnelle du devenir historique ${ }^{8}$.

Récit de vie ou récit du monde, l'utilisation du « je " est tabou mais c'est ce qui «fait passer du mode objectif au mode réflexif, du vérisme hyalin au récit d'enquête, de l'impersonnalité académique au carnet de recherche, à l'autobiographie critique " (Jablonka, 2014 : 290). Et c'est à partir de ce constat qu'on va étudier Mes soixante ans, en tant que témoignage personnel du fait historique.

\footnotetext{
${ }^{8}$ Le genre de cet ouvrage est également étudié par Ángela Magdalena Romera Pintor (2015).
} 


\section{La Littérature aux prises avec la méthodologie historique}

Dans tout travail de recherche en Histoire le moi du chercheur remplit trois fonctions. Premièrement, le « je " de position qui indique la situation de chercheur : il s'agit de se définir par rapport au contexte socio-politique, de préciser les motivations et les filiations ainsi que de déterminer un système de valeurs. Deuxièmement, le " je » d'enquête qui déploie le raisonnement et les argumentations de recherche: décrire l'expérience, avancer des preuves, faire proliférer ou détruire les hypothèses et se justifier. Troisièmement, le "je " d'émotion qui témoigne d'un cheminement : faire état des infra-découvertes, des surprises, des bouleversements intérieurs qui, certes, influencent le résultat final. Faire abstraction de cette instance de production, considérer l'énoncé objectivement tout en négligeant la part de subjectivité du chercheur, serait se priver d'une partie de la science (Jablonka, 2014 : 290).

Ainsi, lire un manuel d'Histoire exige cette prise de conscience que ce qu'on découvre n'est pas l'Histoire, la réalité pure et dure, mais c'est le chercheur et son point de vue sur un objet. L'énonciateur est aussi important, et même plus que l'énoncé. Notre attente face à un ouvrage d'écriture du soi qui prétend créer des connaissances sur la réalité du monde, la présenter, l'expliquer et la faire mieux comprendre aux autres, devrait être modulée de la même façon que face à un ouvrage scientifique. Voyons maintenant comment opèrent ces trois « je " de la méthodologie historique dans Mes soixante ans.

\subsection{Le « je» de position}

Femme poète républicaine : dès l'épigraphe, Salm affiche son identité de poétesse inspirée et son attachement aux valeurs de la Révolution. Elle sollicite les muses pour qu'elles associent leurs voix à la sienne afin de retracer les moments magnifiques de la Révolution de 1789, " ces temps tumultueux " et pourtant "glorieux ", dont le souvenir revivifie son talent créateur tout au long de sa vie :

Muses! redisons-les ces temps tumultueux

Dont la seule pensée et m'enflamme et m'oppresse ;

Ces temps de gloire, de détresse,

Qui seront admirés par nos derniers neveux ;

Muses! redisons-les! Au déclin de ma vie,

Lorsque des ans encore je brave les rigueurs,

Muses, que je consacre à ma noble patrie,

A son double réveil ce reste d'énergie ;

Muses! redisons ses grandeurs (Salm, $1842: 270-71)^{9}$ !

Inspiratrices du poète - toujours incarné dans un corps masculin - les Muses sont toutefois invoquées ici pour inspirer la femme poète. Ces vers mis en épigraphe

\footnotetext{
9 Toutes les références sont données à la dernière édition du texte publiée du vivant de l'écrivaine en 1842, qui se trouve numérisée sur le site de Gallica: https:/gallica.bnf.fr/ark:/12148/bpt6k204684m.r=Constance\%20de\%20salm\%20oeuvres\%20compl\%C3\%A8tes?rk=21459;2.
} 
rappellent aussi la lutte de Constance de Salm pour faire reconnaître son statut de femme poète inspirée et pour contester les canons qui n'acceptent les femmes dans le domaine de la poésie que comme inspiratrices ${ }^{10}$.

La double identité de l'écrivaine est aussi révélée dans les premiers vers du texte, lorsque pour justifier l'audace de parler de soi, Salm n'avance ni l'argument du témoignage historique ni celui de servir "la cause de l'humanité " comme disait Germaine de Staël. Angoissée par les atteintes de l'âge, elle écrit le récit de sa vie pour se rassurer sur la vivacité de sa capacité créatrice. Elle fouille donc sa mémoire et interroge sa source d'inspiration :

Le voilà donc ce triste hiver de l'âge

Que, jeune, je croyais le moment du repos,

De ce repos forcé que doit subir le sage ;

[...]

Ne me trompé-je point pourtant?

Quand aussi sur mon front j'aperçois son ravage,

Mon esprit se sent-il de vains troubles exempt ?

Au mot de gloire, de patrie,

Un généreux, un noble sentiment

En moi s'élève-t-il encore subitement?

$[\ldots]$

L'espoir d'orner mon nom des lauriers du talent,

Est-il toujours l'orgueil, le besoin de ma vie,

Son ivresse de chaque instant?

$[\ldots]$

Oui, je 1e sens, je me sens inspirée ... (Salm, 1842 : 267-68).

Si la notion de gloire englobe son désir de renommée dans le présent et de survie dans la postérité, la mention de la patrie peut suggérer son envie de servir une cause commune par son écriture. En effet, le mot, depuis la Révolution, est chargé d'un sens aussi politique et idéologique qu'affectif; il incarne à lui seul les idéaux de liberté et de bonheur ainsi que le peuple révolutionnaire cherchant dans sa terre natale un facteur d'unification en face des monarques de l'Europe entière (Tulard, 1998 : 1023).

Par ailleurs, du côté des para-textes aussi, l'avertissement et les notes ajoutées aux différentes éditions du texte montrent la volonté de l'auteure de définir sa position dans un contexte politiquement tendu, lequel a d'ailleurs causé la publication différée de l'œuvre. En fait, née en 1767, Constance de Salm a soixante-six ans lorsqu'elle publie en 1833, Mes soixante ans. Quoique ce nombre rond paraisse plus

\footnotetext{
${ }^{10}$ La querelle sur les femmes poètes en 1797, déclenchée par Écouchard-Lebrun dans les pages de la Décade philosophique, pendant laquelle l'écrivaine défend le droit des femmes à l'instruction et à la participation dans la sphère publique, reste le moment le plus important de la carrière littéraire de Constance de Salm.
} 
propre à servir de titre à un ouvrage, Salm a tenu à expliquer ce choix. La première édition du texte contient une note qui éclaire la raison de ce décalage entre l'âge annoncé et l'âge réel de l'écrivaine :

Je venais d'avoir soixante ans (en 1827) lorsque j'ai commencé cet ouvrage. La difficulté d'exprimer alors librement tout ce que j'avais à dire sur les grands événements que je rappelais, me l'avait fait abandonner, et ce n'est que l'année dernière, et après avoir publié mon Épître aux souverains absolus, que je m'en suis occupée de nouveau. Il ne m'a point paru que cette différence de quelques années m'obligeât à renoncer au titre de Mes Soixante Ans auquel se rapportent le début, la fin et même le plan général de ces souvenirs : mais pour l'exactitude des dates, mon âge se trouvant indiqué dans quelques biographies, j'ai cru devoir donner cette explication (Salm, $1833: 72$ ).

Justifier le choix de garder le premier titre en s'appuyant sur la logique interne du texte (" le début, la fin et même le plan général ») ne semble pourtant pas tout à fait exact. Mes soixante ans s'achève sur la Révolution de 1830 lorsque l'écrivaine avait soixante-trois ans et non pas soixante. D'ailleurs, dans une autre note relative à la deuxième édition du texte $(1835)^{11}$, Constance de Salm écrit qu'elle avait pensé continuer le récit de ses souvenirs jusqu'au moment de leur publication, mais que finalement elle y a renoncé :

Lorsque j'ai fait le plan de cet ouvrage, mon intention avait été de ne m'arrêter dans mes récits qu'au moment où je le ferais paraître. J'y avais, en conséquence, parlé avec quelques détails des années écoulées depuis la révolution de 1830 ; mais m’étant aperçue que tout ce que j'ajoutais à ce grand tableau en affaiblissait l'effet, je me suis décidée à supprimer ces nouveaux récits (Salm, $1835: 279$ ).

En fait, c'est faute de pouvoir "s'exprimer librement" qu'elle suspend l'écriture de Mes soixante ans. Évidemment en 1827, trois ans après le sacre de Charles X et la liquidation quasi-totale des acquis de la Révolution de 1789, l'apologie de ses principes et la critique sévère de la Restauration étaient assez délicates, d'autant plus inconcevables que par une nouvelle ordonnance du 24 juin, la censure venait d'être rétablie. Cette ordonnance créait un bureau de censure et un conseil de surveillance de la censure et envisageait des dispositions si sévères que sur les six censeurs trois refusèrent ce poste. Ces mesures suscitèrent la réaction de Chateaubriand qui écrivit $D u$ rétablissement de la censure par l'ordonnance du 24 juin

${ }^{11}$ La deuxième édition du texte est parue dans le second tome des Ouvrages en prose suivi de Mes soixante ans, 207-278. 
1827. Plutôt que de pratiquer une autocensure massive de ses pensées et de ses sentiments, l'écrivaine préfere abandonner - provisoirement du moins - son projet.

Dans tous les cas, autant dans le texte que dans les para-textes, Salm procède à une opération de contextualisation de soi. Notons d'ailleurs qu'un tel souci d'exactitude révélerait également l'attitude du chercheur qui définit sa position face à son objet, qui se met dans le contexte pour mieux montrer l'orientation de sa pensée ainsi que sa méthode.

\subsection{Le « je » d'enquête}

" N'avancer rien sans preuve" (Bayle, 1720 :1077) est le cœur de la méthode historique. L'intelligibilité et la fiabilité d'un récit sur l'Histoire sont avant tout fondées sur les sources dont le chercheur se sert ainsi que sur les preuves qu'il accumule. En ce qui concerne la source, la réminiscence du passé à la lumière du savoir présent est la principale source de Mes soixante ans. Femme, écrivaine, héritière des Lumières, salonnière, princesse d'Empire, obligée de vivre en Rhénanie allemande, elle a, de la sorte, parcouru une période mouvementée de l'Histoire et possède donc une sorte de profonde connaissance préalable de la matière qu'elle traite, ou comme dit Alain Corbin, elle dispose de ses " archives intérieures» (Corbin, $2006: 6$ ).

L'utilisation fréquente du " je " (presque 150 fois) suivi des verbes de perception, d'argumentation et d'évaluation témoigne de ses tentatives pour donner une image aussi claire que juste de l'Histoire. Elle revit et fait revivre ce qu'elle-même et les autres, morts ou vivants, ont vécu. Le « je » cède fréquemment la place au " on » et $\mathrm{au}$ "nous" (au total environ 90 fois, corrélés tantôt au peuple français tantôt aux Françaises seules). Il s'agit donc de présenter et d'expliquer un certain nombre d'expériences, de sentiments et de souvenirs communs. Le texte comme d'autres mémoires vise « explicitement à construire une représentation des événements passés sur laquelle puissent s'accorder les lecteurs contemporains et leurs successeurs et à ce titre, il offre un véritable cadre de remémoration partagée " (Jannelle, $2012: 3$ ). La capacité de co-sentir étant " un maillon essentiel de connaissance [selon] Ricœur, Corbin, Monod [historien français], Dilthey [historien allemand] " (Jablonka, 2014 : 168), le recours aux souvenirs collectifs pour corroborer les faits et les jugements serait donc fiable.

Par ailleurs la preuve n'est pas préétablie et restrictive ; «il n'y a pas de source en soi, il y a seulement des objets qu'on attire, qu'on transforme en preuve, par le regard que l'on porte sur eux. Inversement, n'importe quel objet peut servir de preuve, pourvu qu'on ait l'intention de démontrer quelque chose avec " (Jablonka, 2014 : 174). De ce fait, chaque fois que Salm évoque les institutions politiques et juridiques ou qu'elle rappelle les idées philosophiques et les idéaux révolutionnaires, elle avance méthodologiquement ce qu'Aristote (2007 : 76), dans sa Rhétorique, appelle des preuves extra-techniques : 
Entre les preuves, les unes sont extra-techniques, les autres techniques ; j'entends par extra-techniques, celles qui n'ont pas été fournies par nos moyens personnels, mais étaient préalablement données, par exemple, les témoignages, les aveux sous la torture, les écrits, et autres du même genre ; par techniques, celles qui peuvent être fournies par la méthode et nos moyens personnels; il faut par conséquent utiliser les premières, mais inventer les secondes.

Le rappel de la "tribune " au premier temps de la Révolution, peut être une référence aux assemblées des états généraux ou de la Constituante et un signe du changement du régime politique: "Que l'on se trouvait grand lorsque la voix du sage/ Du haut de la tribune éclairait l'univers» (Salm, 1842 : 272). L'évocation de la nouvelle organisation du pouvoir politique sert aussi de preuve pour distinguer l'Ancien Régime de la modernité politique :

Quand, d'une nation partageant les transports,

On croyait presque sans efforts

Entre tous les pouvoirs établir l'équilibre,

Et par de nouveaux droits effacer de vieux torts (Salm, 1842:271).

Faut-il voir là une allusion à la théorie de la séparation des pouvoirs de Montesquieu, à l'article seize de la Déclaration des droits de l'homme qui affirme que «Toute société dans laquelle la garantie des Droits n'est pas assurée, ni la séparation des pouvoirs déterminée, n'a point de constitution " ou au titre trois de la Constitution de 1791 (des pouvoirs publics) qui aspire à consolider la souveraineté nationale et la division entre les pouvoirs législatif, exécutif et judiciaire?

Faisant l'éloge de Napoléon soutenu alors par les savants et les artistes (allusion aux Instituts), Salm rappelle tour à tour les tentatives d'unifier juridiquement la France (évocation du Code Civil), de construire des édifices géants en vue de perpétuer le souvenir des victoires de l'armée française (comme l'arc de triomphe) et de stabiliser la société par la réconciliation avec le catholicisme (souvenir du Concordat) :

S'entourer de savants, d'artistes, de guerriers,

Frapper l'improbité, rechercher le mérite,

Attacher son grand nom au code, aux monuments,

Relever les autels en traçant leur limite,

Honorer les mœurs, les talents (Salm, $1842: 291)$.

Soit par leurs convictions sincères, soit en raison des exigences propagandistes des gouvernements, tous les artistes furent alors poussés à mettre en ouvre l'actualité ou l'idéal politique ; écrivant "Quand le peintre, en son beau délire, / Retraçait le haut fait qui l'avait transporté " (Salm, 1842 : 282) elle fait évidemment allusion au peintre David dont la mort de Marat et le sacre de Napoléon, tout comme la liberté 
guidant le peuple d'Eugène Delacroix, sont inséparables de l'imaginaire collectif de la première moitié du XIX siècle. La Marseillaise et la cocarde tricolore sont également d'autres emblèmes de la République évoqués lors de la mémoration de la révolution de 1830 :

Grâce te soit rendue, ô destin généreux

Qui, sur le déclin de ma vie,

Encore as permis que mes yeux

Revissent ces couleurs, symbole de la gloire ;

Qu'encore mon oreille entendît

Ce chant de liberté proscrit (Salm, $1842: 324)$.

S'ajoutent à ces références aux institutions, aux objets, voire aux personnes, celles aux idées et aux principes fondateurs de la France régénérée : la liberté, la raison, la philosophie, l'égalité, la citoyenneté, la république, la nation et la patrie; les mots portent de lourdes charges symboliques dès le lendemain de la Révolution.

La liberté paraît dans Mes soixante ans le principe primordial qui distingue la France post-révolutionnaire de l'Ancien Régime. La liberté de l'individu, la liberté de la nation et celle de la circulation des idées sont les aspects divers d'un principe de la République singulièrement cher à Constance de Salm. Évoquée d'abord, aux côtés de " la clarté nouvelle », de la " civique vertu » et de la "sage égalité » (Salm, 1842 : 269) pour singulariser la France régénérée, par la suite "l'auguste liberté » surgit seule (et souvent au pluriel) pour récapituler tous les autres principes.

Le premier épisode qui traite de la Révolution s'achève sur l'affirmation des " pactes et des serments" entre le peuple et le pouvoir qui seront " Du despotisme la barrière, / et de nos libertés le principe et l'appui "(Salm, 1842:272)! À la fin du Directoire, pour glorifier la France et le succès des Français, sont saluées aussi bien les victoires de l'armée que les « libertés »:

Sûre encore de ses libertés,

Sans cesse triomphante et sans cesse agrandie,

Partout elle portait ses armes, ses clartés (Salm, 1842 : 282).

Sous l'Empire et la Restauration encore, c'est la liberté qui rappelle la Révolution de 1789, ses acteurs et ses acquis et la révolution de 1830 est aussi saluée pour sa reconquête de la liberté :

Et dans tout son éclat, toute son énergie,

La liberté superbe, imposante, agrandie,

Sous un roi citoyen promettant le bonheur;

La liberté conquise par l'honneur (Salm, 1842 : 322).

La libre expression de la pensée constitue un autre aspect de la liberté à laquelle est attachée l'écrivaine qui déplore que la censure napoléonienne l'ait restreinte. La dénonciation de la censure se transforme en profession de foi sous la 
plume d'une femme qui attribue à la littérature le pouvoir d'influencer les esprits et d'orienter les vœux du peuple :

Le grand vainqueur, osons le dire,

Redoutait de la vérité

La lumière, la force et la sévérité ;

Dans ses vastes desseins craignant de laisser lire,

Le droit de parler et d'écrire

Sur le présent, l'avenir, l'alarmait ;

Son esprit juste lui disait

Qu'un sage écrit dure plus qu'un empire ;

Il observait les lettres qu'il flattait ;

Par leur puissance en secret oppressée,

Son âme enfin s'en irritait,

Et ne pouvant étouffer la pensée,

Sa main de fer la comprimait (Salm, $1842: 300$ ).

Les Français ne sont plus de simples sujets des rois, ils sont des citoyens " égaux et frères" (Salm, 1842: 272). Si les mots évoquant la "citoyenneté " (citoyen, civique) figurent fréquemment dans le poème, le mot de nation (souvent "grande") se répète aussi abondamment. La Nation incarne l'ensemble des liens complexes entre les individus et fonde entre eux le sentiment d'une appartenance commune. Ils s'y reconnaissent dans un corps unique avec la volonté durable de le protéger et de protéger ses valeurs, ils y trouvent aussi l'emblème de leur pouvoir politique, comme le dit l'article trois de la Déclaration des droits de l'homme et du citoyen: "Le principe de toute souveraineté réside essentiellement dans la Nation. Nul corps, nul individu ne peut exercer d'autorité qui n'en émane expressément ». À l'occasion des fêtes révolutionnaires, Salm (1842:278) écrit :

Quand je chantais enfin la grande nation,

La grande nation dans les siècles célèbre,

Oubliant ses malheurs pour soutenir ses droits;

Que l'on venait de voir sous le crêpe funèbre,

En imposer encore aux despotes, aux rois ;

La grande nation de gloire environnée,

Alors sans alliés, sans appui, sans secours,

Bravant les factions, l'Europe déchaînée,

S'égarant quelquefois, mais s'illustrant toujours.

Si les objets matériels, les documents, les concepts philosophiques font ainsi communiquer le poème - le texte esthétique - avec le hors-texte - le Réel - et rendent par conséquent possible une lecture scientifique du texte, le comparatisme est un autre pilier de la méthode historique auquel a recours l'écrivaine pour avancer son argumentation. Pour être capable de raconter l'Histoire, il est essentiel de "s'ouvrir à d'autres périodes, d'aller et venir entre passé et présent, de circuler à travers le 
monde " (Jablonka, 2014 : 171). La capacité de confrontation, le souci de ne pas s'en tenir à son seul sujet ni de l'idolâtrer garantissent la scientificité de la démarche et du résultat final.

La nostalgie envers les "temps de grandeur et gloire » de la décennie révolutionnaire que Salm regrette et l'accent mis sur leur différence avec «ceux qu' [elle voit] aujourd'hui » dominent Mes soixante ans. Chaque épisode de l'histoire est comparé à la période révolutionnaire, au cours de laquelle l'écrivaine avait vu la réalisation de ses idéaux. Les critères tirés de cette période sont les facteurs d'évaluation des gouvernements successifs. Le témoignage historique cède ainsi la place au jugement de valeur et le texte nous permet à la fois de capter le regard de l'historienne et d'examiner ses convictions idéologiques.

Le retour des manières de l'Ancien Régime sous l'Empire est retracé lorsque l'écrivaine évoque « la soif des titres » ou " le luxe, le flatteur, l'or, l'hôtel magnifique " $(1842: 297)^{12}$ :

Tout devint noble et fier, tout devint sombre et triste ;

Tout fut classé ; le grand, l'homme en place, l'artiste.

Ils n'étaient plus ces heureux temps

Où les lettres, où les talents,

Sur les titres, les noms remportaient la victoire (Salm, 1842 : 298).

L'enthousiasme de Constance de Salm face à la décennie révolutionnaire, caractérisée par l'espoir, la raison, le bonheur et l'honneur, se confirme lorsqu'elle décrit la Restauration comme une décadence :

Ah! sans doute, des maux que peut souffrir l'honneur,

Il n'en est point de plus terrible.

Mais voir sur de vieux préjugés

S'établir la vieille puissance ;

Par la nullité, l'ignorance,

Voir le droit, le mérite et la force outragés ;

Voir méconnaitre avec audace

Les lois et la foi des serments ;

Voir une cour, un roi s'occuper d'une chasse,

Quand la honte menace au dehors, au-dedans ;

Voir l'erreur, la sottise altière,

Sans cesse élever leur barrière

Entre elles et la vérité,

Et forcer enfin la lumière

À reculer devant l'obscurité (Salm, 1842 : 305).

\footnotetext{
${ }^{12}$ Reste à mesurer la sincérité de Salm qui, sous l'Empire, s'est ralliée au régime napoléonien et a même écrit une Épître à l'empereur Napoléon en le comparant avec Achile! Cette question est aussi abordée par Romera Pintor (2015).
} 
L'affirmation de la suprématie de l'armée française sous la Révolution et l'Empire, et par comparaison le constat des défaites de la fin de l'Empire et de la Restauration, permettent de voir dans les prises de positions de l'écrivaine sinon un nationalisme radical du moins un patriotisme convaincu. En 1833, elle salue toujours les guerres révolutionnaires et glorifie la France qui, s'emparant d'autres pays, étend aussi bien son territoire que ses idéaux :

Sans cesse triomphante, sans cesse agrandie,

Partout elle portait ses armes, ses clartés

Elle faisait une même patrie

Des riches bords du Rhin, de la belle Italie (Salm, 1842 : 282).

Le ralliement de Constance de Salm à la politique de guerre des gouvernements français apparaît encore lorsqu'elle regrette, en évoquant l'occupation de la France, le temps où les pays " des bords de l'Elbe aux bords du Tibre " faisaient partie de la France, qui n'est désormais plus libre que " dans ses étroits remparts " (Salm, 1842 : 316). Elle fait ici allusion au Congrès de Vienne dont l'acte final ramène la France à ses frontières de 1791 .

Ainsi, quoique le texte soit sous le signe de la poésie, de l'émotivité, le projet de donner une image juste qui empêche d'oublier une France révolutionnée par la philosophie et moderne par ses nouvelles institutions politiques, l'envie de peindre le Réel et de dire le vrai, poussent l'écrivaine à recourir inconsciemment aux méthodes historico-scientifiques.

\section{3. Le « Je » d'émotion}

Constatons d'emblée que Constance de Salm est une écrivaine formée dans la pure tradition didactique des Lumières et la poésie est pour elle, comme elle l'était pour Voltaire, une argumentation versifiée. Le « je » chez elle, est " un je résolument référentiel " (Planté, 2010 : 32) qui évoque les personnes et les événements. Elle envisage le langage poétique "comme traduction, vêtement, ornement - ou travestissement - d'une pensée préalable qu'il s'agit de retranscrire au plus juste " (Planté, 2010 : 19). Toutefois parler de la poésie c'est rencontrer l'émotion profonde qui ébranle le cœur de l'artiste. Mes soixante ans est constitutivement sous le signe de la littérature et ce n'est qu'accessoirement qu'on le lit d'un regard historique. Donc qu'il y ait, dans ce poème, de l'émotion, de l'ébranlement joyeux ou de la mélancolie semble évident.

S'affirmant redevable de l'enthousiasme de la période révolutionnaire, elle atteste elle-même que l'Histoire lui était une source d'inspiration poétique :

C'est que le transport qui m'enflamme

En moi s'est retrempé dans ces temps de grandeurs,

D'enthousiasme, de terreurs,

[...]

C’est que par ces hautes pensées 
Sans cesse ranimant mes esprits et mes sens,

Je vis, j'existe encore dans ces splendeurs passées

Que ne peut atteindre le temps (Salm, 1842 : 269).

La séquence conclusive du poème - la conclusion n'est-elle pas l'aboutissement d'une réflexion scientifique? - est écrite sous le signe du regret : alors que la France, sous la règne du "roi-citoyen " Louis-Philippe I ${ }^{\mathrm{er}}$, retrouve l'espoir d'incarner les idéaux de 1789, l'écrivaine avance dans l'âge et craint de ne plus voir l'accomplissement de ses vœux de liberté :

OUI! le monde verra cette grande lumière ;

Mais dans tout son éclat, hélas!

Dans toute sa splendeur, je ne la verrai pas.

[...]

Et l'âge vient, et j'aperçois

Déjà la fin de ma carrière :

Je ne le verrai pas le triomphe des droits,

Il n'enchantera point ma course passagère ;

Mais je saurai qu'un jour il brillera,

Que chaque instant nous en rapprochera,

Et je pourrai me dire à mon heure dernière :

L'œuvre de la justice enfin s'accomplira (Salm, 1842 : 328).

Sa position de républicaine accentuée par celle de poétesse, mettent inévitablement la réminiscence du passé sous le signe de l'émotivité. Prenons l'exemple du passage où Salm se souvient de la querelle des femmes poètes et reprend la défense des droits de son sexe à l'écriture de la poésie. S'opposant à l'infériorité imposée aux femmes, elle argumente en terme politique :

Nous devions végéter dans un obscur état;

Despotes du Parnasse, ils y faisaient renaître

Ces féodales lois que leur raison brisait,

Et nous devions subir les caprices du maître,

Quand à ce mot encore la France frémissait (Salm, $1842: 279)$.

Si les hommes poètes ne tolèrent que difficilement, et de façon exceptionnelle, les femmes poètes, s'ils voient en elles des rivales et les condamnent à l'invisibilité, Constance de Salm voit dans leur attitude les mêmes rapports de domination qui existaient dans la société civile. Elle dénonce la volonté masculine de cantonner les femmes dans la sphère privée. Par l'emploi des mots "despotes ", "lois féodales ", "maître ", elle lie son sujet à l'histoire vécue de la France et insiste sur la charge politique de la querelle des femmes poètes. La modernité de sa pensée réside dans le fait qu'elle a bien saisi le caractère politique de la domination masculine. Tout cela n'empêche pas pour autant son emportement envers les hommes qu'elle qualifie de «jaloux ", d' "ingrat[s] " ou d' " orgueilleux". Si en 1797, dans l'Épître aux 
femmes, elle écrivit "L'homme injuste, jaloux de tout assujettir, / Sous la loi du plus fort prétend nous asservir » (Salm, 1842: 6), en 1833 elle réitère :

Tout à coup on vit le jaloux,

Du haut de sa grandeur factice,

Au saint nom du devoir s'élever contre nous.

Dans son orgueilleuse colère,

L'ingrat, il outrageait ce sexe qui naguère

A l'homme prodiguait de si tendres secours;

Ce sexe si sublime et si grand dans ces jours

Où le sang inondait la terre (Salm, $1842: 278$ ).

La "colère ", même si Virginia Woolf la juge nuisible à la qualité de l'argumentation, motive l'écrivaine qui depuis 1794 (tragédie-lyrique de Sapho), dénonce les différentes formes du patriarcat aussi bien dans la sphère publique que privée. L'argumentation politique n'exclut point l'émotion.

Poétesse inspirée elle ne dissimule ni sa sympathie ni son antipathie, pourtant cela n'influence guère sa volonté de justesse et d'impartialité pour avancer ses jugements (l'éthique de recherche). Le rappel des « discordes civiles» au premier moment de la Révolution paraît d'abord contradictoire avec les vers qui viennent juste d'insister sur l'unanimité et la fraternité des Français, mais il montre la volonté de transmettre une image fidèle des événements qui ont marqué la France et l'esprit de l'écrivaine. Le même souci d'objectivité se révèle dans l'épisode de la Terreur où malgré l'attachement que l'écrivaine porte à la République, le régime de Robespierre est décrit comme un "civisme en fureur." Malgré toutes les allusions aux bienfaits de l'Empire, il n'échappe pas à l'écrivaine qu'il s'agit d'un régime despotique : elle qualifie Napoléon de "déserteur de la liberté " et écrit en majuscules que "LA GRANDE NATION, / TRIOMPHANTE PARTOUT, EST CHEZ ELLE VAINCUE » (Salm, 1842 : 295). La majuscule parait aussi le signe graphique du cri d'alarme et de détresse que Salm souhaite faire entendre.

\section{Conclusion}

Si le but de toute démarche scientifique est d'enrichir la connaissance humaine, la littérature du Réel qui dévoile la vérité sur le monde et le moi, pourra se doter du statut scientifique. Quand Salm (1842: 288) écrit : "Qu'il m'est doux de les peindre encore / Ces jours qui ne reviendront plus ; / [..] / Ces jours qu'on méconnaît parce qu'on les ignore", elle affiche l'ambition de peindre l'inconnu, d'élucider en détail le factuel non exploité et resté incompris ; cela montre bien son désir d'ajouter aux connaissances du monde. Sa démarche est celle d'Annie Ernaux qui, presque deux siècles après, l'explique en ces mots :

[...] ce que représente écrire pour moi [est] descendre dans la réalité de ce que nous avons vécu d'une façon collective mais au travers de ce que j'ai vécu personnellement. Il y a dans le vé- 
cu quelque chose d'immense, qui demande à être questionné sans cesse. C'est cela l'écriture qui vise à la connaissance, qui exsude la connaissance du vécu et du réel (Ernaux, 2014 : 83).

La prise de distance par rapport aux faits et aux expériences passés, leur explication à la lumière des informations futures, tout autant que l'agencement chronologique du réel, l'organisation taxologique de la réalité selon des catégories historiques, sont des attitudes scientifiques. D'ailleurs, doter le texte d'une introduction et d'une conclusion, comme aussi recourir à diverses preuves pour argumenter en faveur des principes philosophiques ou pour accentuer le témoignage historique, concourent à renforcer la qualité scientifique du texte. Quoique Salm ne pense pas écrire de l'Histoire dans Mes soixante ans, retracer ses souvenirs et partager ses impressions la conduisent à laisser une version de l'Histoire de la France vue et vécue par elle et ses contemporains. Ce faisant, pour appuyer la justesse de son témoignage et de ses jugements, elle argumente insciemment avec les outils de la méthodologie historique et fait de la littérature un outil d'explication-compréhension du monde, un texte apparenté à un raisonnement.

\section{RÉFÉRENCES BIBLIOGRAPHIQUES}

ABRANTÈS, Laure Junot (1831) : Mémoires de Madame la duchesse d'Abrantès, ou Souvenirs historiques sur Napoléon : la Révolution, le Directoire, le Consulat, l'Empire et la Restauration, Paris, Ladvocat.

ADELSON Robert \& Jacqueline LETZTER (2000) : « French women opera composers and the aesthetics of Rousseau ». Feminist Studies, 26/1, 69-100.

ARISTOTE (2007) : Rhétorique, Livre I, trad. Pierre Chiron, Paris, Flammarion.

BAYLE, Pierre (1720) : "Épicure ", in Dictionnaire historique et critique. Roterdam, Bohm, vol. $2,3^{\text {e éd. }}$

Berenguier, Nadine (2017) : «Publish or Perish : Constance de Salm’s Identity Crisis and Unfulfilled Promise ». Dix-Neuf Journal of the society of Dix-Neuviémistes, 21/I, 4668

Berenguier, Nadine (2017) : "The Expatriate and the Traveler: Constance de Salm and Germaine de Staël in Germany ». Women in French Studies, 7, 138-163.

BOURDIEU, Pierre (1982) : Leçon sur la leçon. Paris, Minuit.

COnstant, Benjamin (1992) : Portraits, mémoires, souvenirs. Textes établis et annotés par Éphraïm Harpaz. Paris, Honoré Champion.

CORBIN, Alain (2006) : "Ne rien refuser d'entendre ». Entretien avec Alain Corbin ; propos recueillis par Vincent Casanova, Philippe Mangeot et Philippe Masanet. Vacarme, 35/2, 4-12. Disponible sur: https://www.cairn.info/revue-vacarme-2006-2-page4.htm\#. 
Crogiez Labarthe, Michèle (2010) : "Mes soixante ans : la gloire au féminin ". Cahiers Roucher-André Chénier, 29, 103-113.

ERNAUX, Annie (2008) : Les Années. Paris, Gallimard.

ERNAUX, Annie (2011) : Écrire la vie. Paris, Gallimard.

ERnAuX, Annie (2014) : Le vrai lieu. Entretiens avec Michel Porte. Paris, Gallimard (coll. Blanche).

Fend, Mechthild, Melissa Hyde \& Anne LAFOnT [dir.] (2012) : Plumes et pinceaux: Discours de femmes sur l'art en Europe (1750-1850). Dijon, Les Presses du réel.

FRAISSE, Geneviève (1989) : La Muse de la raison: la démocratie exclusive et la différence des sexes. Aix-en-Provence, Alinéa.

GENETTE, Gérard (2004) : Fiction et diction. Paris, Seuil.

GUSDORF, George (1991) : Lignes de vie, 1. Les écritures de moi. Paris, Odile Jacob.

HARTOG, François (2012) : "Ce que la littérature fait de l'histoire et à l'histoire ». Fabula / Les colloques: Littérature et histoire en débats. Disponible sur : http://www.fabula.org/colloques/document2088.php.

JABLONKA, Ivan (2014) : L'Histoire est une littérature contemporaine. Manifeste pour les sciences sociales. Paris, Seuil.

JEANNELLE, Jean-Louis (2012) : "Les Mémoires, impensé générique des guerres de mémoires ». Fabula / Les colloques: Littérature et histoire en débats. Disponible sur: http://www.fabula.org/colloques/document2092.php.

LE BozeC, Christine (2019) : Les femmes et la Révolution 1770-1830. Paris, Passés Composés.

LeJEUne, Philippe (1998) : L'autobiographie en France. Paris, Armand Colin.

LETZTER, Jacqueline (1999): " Making a spectacle of oneself : French revolutionary opera by women ». Cambridge Opera Journal, 11/ 3, 215-232.

LuZON, Martine (1997): Une mémorialiste féministe : Constance de Salm. Mémoire de mâ̂trise ès Lettres. Montréal, Université McGill.

MATTOS, Rudy Frédéric (2007) : The discourse of Women Writers in the French Revolution: Olympe de Gouges and Constance de Salm. Dissertation in partial fulfillment of the requirements for de degree of doctor in Philosophy. Austin, University of Texas.

PAQUIN, Éric (1998) : «Du paratexte au texte : des défis formel. La poétique épistolaire de Constance de Salm ", in Le récit épistolaire féminin au tournant des Lumières et au début du XIXe siècle (1793-1837): adaptation et renouvellement d'une forme narrative. Thèse de doctorat en étude français. Montréal, Université de Montréal, 130-162.

PERAZZOLO, Paola (2018): «Un "animal sans pareil” sous la Révolution : la Sapho ambiguë de Constance de Salm ». Revue italienne d'études françaises, 8. DOI : https://doi.org/10.4000/rief. 1773 .

Planté, Christine (1989) : "Constance Pipelet : la Muse de la Raison et les despotes du Parnasse », in Les femmes et la Révolution française. Toulouse, Presses Universitaires du Mirail, 285-295. 
PlanTÉ, Christine (2010) : "Muse de la Raison, et poète ? ». Cahiers Roucher-André Chénier, 29, 13-40.

PlANTÉ, Christine (2012): "Un roman épistolaire féminin? Pour une critique de l'imaginaire générique: Constance de Salm, Vingt-quatre heures d'une femme sensible ", in C. Mariette-Clot \& D. Zanone, La Tradition des romans de femmes. XVIII ${ }^{e}-$ XIX siècles. Paris, Champion, 275-296,

REID, Martine (2012) : Des femmes en littérature. Paris, Belin.

ROMERA PINTOR, Ángela Magdalena (2015a) : Constance de Salm y la modernidad de su discurso feminista. Epistolas y otros escritos (1767-1845). Valence (Espagne), Publicacions de la Universitat de València.

ROMERA PINTOR, Ángela Magdalena (2015b) : « Mes soixante ans y sus referentes políticos y literarios: las memorias en verso de Constance de Salm ». Çédille, revista de estudios franceses, 11, 439-467. Disponible sur: http://cedille.webs.ull.es/11-DEF/20romera.pdf.

SALM, Madame la Princesse Constance de (1833): Mes soixante ans ou Mes souvenirs politiques et littéraires, Paris, Arthus Bertrand, Firmin Didot frères, Delaunay, PalaisRoyal.

Salm, Madame la Princesse Constance de (1835): Ouvrages en prose suivi de Mes soixante ans. Paris, Firmin Didot frères.

Salm, Madame la Princesse Constance de (1842) : Euvres Complètes. Paris, Firmin Didot frères.

SHARIF, Maryam (2014): Constance de Salm (1767-1845): une modernité contradictoire. Thèse de doctorat ès Lettres et art. Lyon, Université Lumière Lyon 2.

SHARIF, Maryam (2015) : "La Révolution française et le Théâtre de propagande : exemple de Sapho (1794) de Constance Pipelet ». [Recherche en langue et littérature françaises (Presses de l'Université de Tabriz)], printemps-été, 43-74.

SETH, Catriona (2010a) : "L'Épître aux femmes: textes et contextes ". Cahiers Roucher-André Chénier, 29, 41-64.

SETH, Catriona (2010b) : "La femme auteur, stratégies et paradigmes. L'exemple de Constance de Salm ", in A. del Lungo \& B. Louichon (dir.), La littérature en bas-bleus Romancières sous la Restauration et la Monarchie de Juillet (1815-1848). Paris, Classique Garnier, 195-213.

STAËL, Germaine de (1996) : Dix années d'exil. Édition critique par Simone Balayé et Mariella Vianello Bonifacio. Paris, Fayard.

Thion Soriano-Mollà, Dolores, Noémie FrançOIS \& Jean AlbresPit (2016) : Fabrique de vérité(s) vol. 2. L'ouvre littéraire au miroir de la vérité. Paris, L'Harmattan.

TULARD, Jean (1998) : Histoire et dictionnaire de la Révolution française 1789-1799, Robert Laffont.

VIENNOT, Éliane (2016) : Et la modernité fut masculine. La France, les femmes et le pouvoir. Paris, Perrin.

ZANONE, Damien (2006) : Écrire son temps, les mémoires en France de 1815 à 1848. Lyon, Presses Universitaires de Lyon. 